\title{
Clinical presentation, diagnostic approach, and treatment of hand lipomas: a review
}

\author{
Sabrina Lichon ${ }^{1}$, Amor Khachemoune ${ }^{2 \bowtie}$
}

\begin{abstract}
Lipomas are the most common soft tissue tumors of mesenchymal origin. They can appear anywhere on the body, and in rare cases they may present on the hands. Lipomas represent approximately $1 \%$ to $3.8 \%$ of all benign tumors in the hands. Typically, lipomas present in the fifth or sixth decade of life, occurring in both men and women. Lipomas may appear anywhere on the hand, often presenting as painless, slow-growing, and mobile masses. Symptoms may develop if local neurovascular structures are compressed. Differential diagnosis includes liposarcoma and fibrolipomatous hamartoma. To assist with clinical diagnosis, ultrasound, computed tomography, or magnetic resonance imaging may be utilized. The best way to confirm diagnosis is with histopathology. The main treatment for hand lipomas is surgical excision. Postoperative recurrence is rare.
\end{abstract}

Keywords: lipoma, hands, treatment

Received: 21 September 2017 | Returned for modification: 26 February 2018 | Accepted: 16 March 2018

\section{Introduction}

Lipomas are the most common soft tissue tumors of mesenchymal origin. They consist of mature adipocytes and can appear anywhere on the body. Most commonly, they occur on the neck, trunk, and upper extremities (1). Lipomas rarely occur in the hands. Lipomas represent approximately $1 \%$ to $3.8 \%$ of all benign tumors presenting in the hands (2).

\section{Epidemiology and etiology}

The etiology of lipomas in general is unknown. Possible theories implicate obesity, trauma, and genetics as contributing to their formation (3, 4). Lipomas are more common in obese individuals (5). Although tumor size may increase with weight gain, tumor size does not decrease during periods of weight loss (3). Two explanations linking trauma to lipoma formation exist. One is that inflammation following trauma may induce lipoma formation through the release of growth factors and cytokines (5). The other is that necrosis of fatty tissue may cause pre-adipocytes to differentiate and form lipomas $(4,5)$. Finally, there may be a genetic component to lipoma formation. Individuals that present with multiple lipomas often report a positive family history (5). Familial cases of angiolipomas, a variant of lipomas, have reported an autosomal dominant inheritance (1). Studies in cytogenetics have linked translocations and inversions involving regions 12q13-15 and 6p13q to lipoma formation anywhere on the body (4).

Lipomas in general often present in the fifth or sixth decade of life $(1,2,5-7)$. However, they may present earlier in life. Neural fibrolipomas, a variant of lipomas, typically present during early childhood or young adulthood (1). Lipomas of the hand occur in both men and women. In their case study of 63 hand lipomas, Carroll and Doyle noted that hand lipomas occurred three times more often in women than in men (8). A possible explanation for this is that women have more adipose tissue (6). However, more recent case studies have shown them to be more common in men $(5,6$, 9).

\section{Clinical presentation}

Lipomas localized to the hand typically present as painless, slowgrowing, mobile masses $(1,2)$. They can appear anywhere on the hand, typically presenting on the thenar and hypothenar eminences (10). Reviews of hand lipomas also include lipomas on the wrist $(5,6)$. Lipomas on the wrist may involve Guyon's canal and carpal tunnel (11). Lipomas occurring on the digits are rare, with an incidence of $1 \%(12,13)$. Although superficial lipomas are more common, hand lipomas often occur beneath the subfascia (1). Typically, lipomas are well-circumscribed and do not invade local structures (11).

Generally, lipomas range from 3 to $5 \mathrm{~cm}$ in diameter. However, hand lipomas are often asymptomatic and may grow to over $5 \mathrm{~cm}$ (2). Lipomas that measure $5 \mathrm{~cm}$ or greater are considered great lipomas, regardless of location (Fig. 1) $(2,7)$.

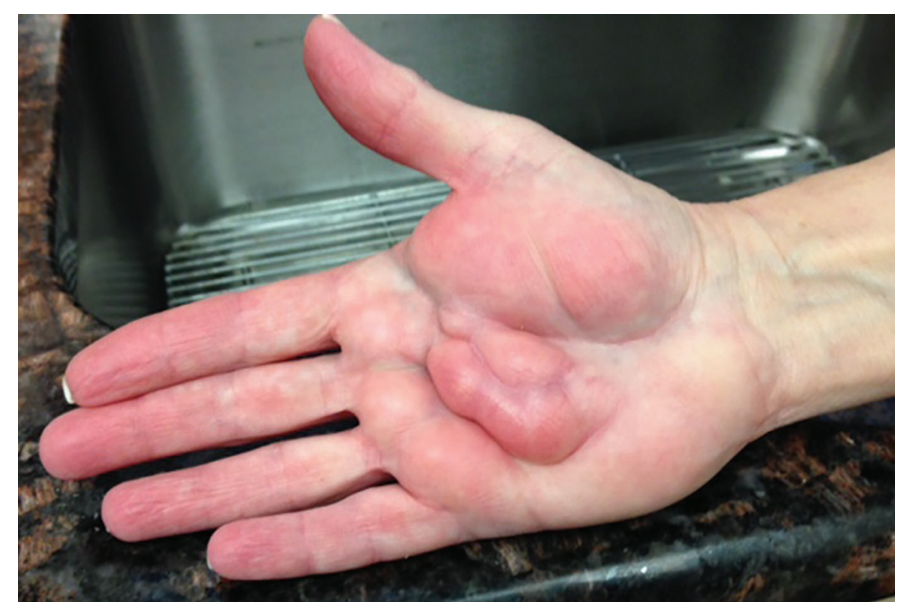

Figure 1 | Rare example of giant palmar lipoma.

Symptoms may arise when the tumor compresses local structures of the hand. For example, local nerve compression may lead to pain, decreased sensation, and decreased motor function (2). Presentation of these symptoms depends on which nerve is compressed. If the median nerve is compressed, this may cause 
carpal tunnel syndrome, whereas a lipoma located in Guyon's canal will cause ulnar hypoesthesia (2).

\section{Histology}

Lipomas are composed of mature adipose tissue separated by thin fibrous septa and blood vessels may be present within the fibrous septa $(1,14)$. Typically, a lipoma is surrounded by a fibrous capsule (14). Other variants of lipomas include neural fibrolipomas, intramuscular and intermuscular lipomas, angiolipoma, and spindle cell lipoma $(1,14)$. Neural fibrolipomas are composed of fibrofatty tissue that surrounds and/or infiltrates local nerves (14). Lipomas that have a mixture of adipose tissue and skeletal muscle are considered intramuscular and/or intermuscular lipomas (1). Angiolipomas show an increased vascular component that can range from 5 to $50 \%$ or more of the tumor (14). Unlike most lipomas, angiolipomas typically present as multiple, painful masses (1). Spindle cell lipomas consist of spindle cells and adipose tissue. Typically, they arise in the subcutis and may extend into the dermis (14).

\section{Differential diagnosis}

The differential diagnosis for lipoma includes liposarcoma and fibrolipomatous hamartoma. Liposarcoma is the second most common soft tissue sarcoma (15). There are different subtypes of liposarcomas, including well-differentiated liposarcoma and myxoid liposarcoma (16). Generally, magnetic resonance imaging (MRI) and biopsy are used to rule out this diagnosis (2). On MRI, liposarcomas tend to appear more heterogenous, poorly encapsulated, and larger in size $(16,17)$. Furthermore, liposarcoma may have thick fibrous septa, nodules, or areas of necrosis on T1-weighted sequences $(10,15)$. On fat-suppressed T2-weighted (FS-T2) or short tau inversion recovery (STIR) images, liposarcomas typically have hypertense septae whereas lipomas have hypointense septae (15). However, well-differentiated liposarcomas may share features of common benign lipomas on MRI (18). If the lesion is larger than 5 $\mathrm{cm}$, the tumor must be considered malignant until proven otherwise (19). Cytogenetics may also aid in diagnosis. One diagnostic characteristic of myxoid liposarcomas is a reciprocal translocation $\mathrm{t}(12 ; 16)$ (q13;p11) (20). Well-differentiated liposarcomas may have cytogenetic abnormalities involving the q13-15 region of chromosome 12; however, this alone is not diagnostic because some benign lipomas have been shown to have this abnormality as well (20).

Fibrolipomatous hamartomas or neural fibrolipomas are benign fibrofatty tumors that arise from the epineurium, making these tumors difficult to resect (2). Similar to lipomas, fibrolipomatous hamartoma in the hands present as slow-growing masses. However, fibrolipomatous hamartomas normally present during childhood or young adulthood, suggesting a possible developmental origin (21). Approximately $80 \%$ of cases involve the median nerve (1). Thus, patients often experience pain, tenderness, paresthesia, decreased sensation, and impaired motor function in areas innervated by the median nerve and its branches $(1,22)$. Involvement of the median nerve may cause carpal tunnel syndrome (2). MRI aids in diagnosis to distinguish between the two. Unlike lipomas, fibrolipomatous hamartomas on MRI have a heterogenous signal due to the mixed composition of fat, fibrosis, and nerve fibers (2). The nerve fascicles typically appear as cylindrical hypointensities (1).

\section{Diagnostic approach}

To assist with clinical diagnosis, ultrasound, computed tomography (CT), or MRI may be utilized. Generally, ultrasound may provide the most rapid assessment and can be used if a patient cannot tolerate an MRI (7). However, CT and MRI provide a more detailed three-dimensional picture of the tumor in relation to local neurovascular structures (2). One study reviewed 134 cases of soft tissue masses in the hand and wrist, and found that the MRI correctly diagnosed $94 \%$ of the cases (23). Although X-rays may be performed, they are not typically useful because the lipoma appears as a homogenous opaque mass (Fig. 2) (2). However, X-rays are beneficial for showing cases of tumor calcification or ossification (2).

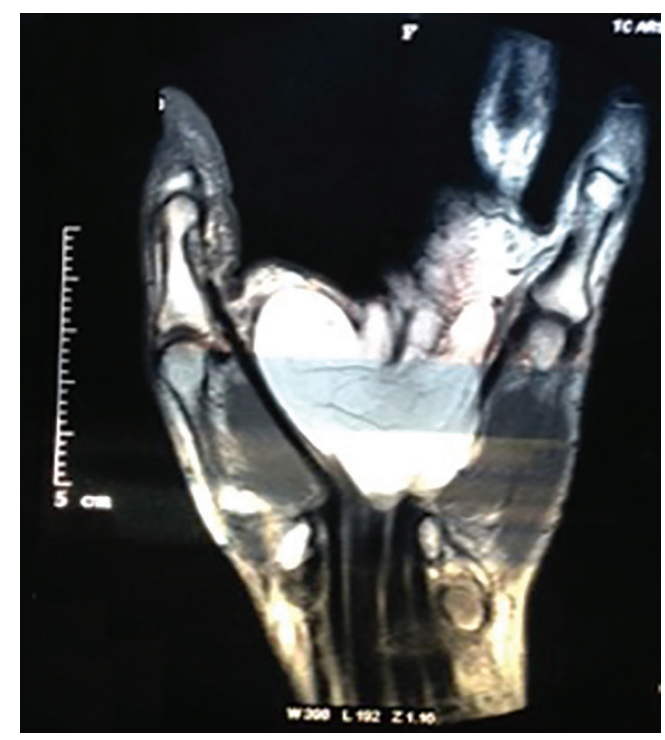

Figure 2 | Example of palmar lipoma on X-ray.

Characteristics to look for on MRI are a thin fibrous capsule, fibrous septa, and fat lobules (24). Lipomas have the same high signal intensity on T1- and T2-weighted MRIs as subcutaneous fat (10). There may be evidence of thin fibrous septa or calcifications, which appear as low signal-intensity areas on T1-weighted and T2weighted sequences (25). A thin fibrous capsule will present as low signal intensity on MRI (1). Although imaging may aid diagnosis, lipomas may share characteristics with liposarcomas (18). The best way to confirm diagnosis is with histopathology.

\section{Medical and surgical management}

The main treatment for hand lipomas is surgical excision (Fig. 3). Patients generally seek treatment due to cosmetic disturbances or functional impairment $(11,16)$. There is a low risk of a lipoma transforming into a liposarcoma (16). Due to the high density of neurovascular structures in the hand, the risk of iatrogenic lesions is high (2). Surgeons must carefully dissect the lipoma from the surrounding structures to ensure complete preservation of motor and sensory function $(2,26)$. In rare cases, complete excision is not performed due to tumor infiltration of local structures $(7,18)$. After surgery, most cases report restoration of sensation and motor function within a year $(4,18,27)$. However, Schmidt recently reported one rare case of radial nerve compression by a giant lipoma that caused an irreparable radial nerve palsy (26). Thus, quick and accurate diagnosis is important for preventing possible irreversible nerve damage. Recurrence of lipomas is rare; however, long-term follow-up is recommended (11). 


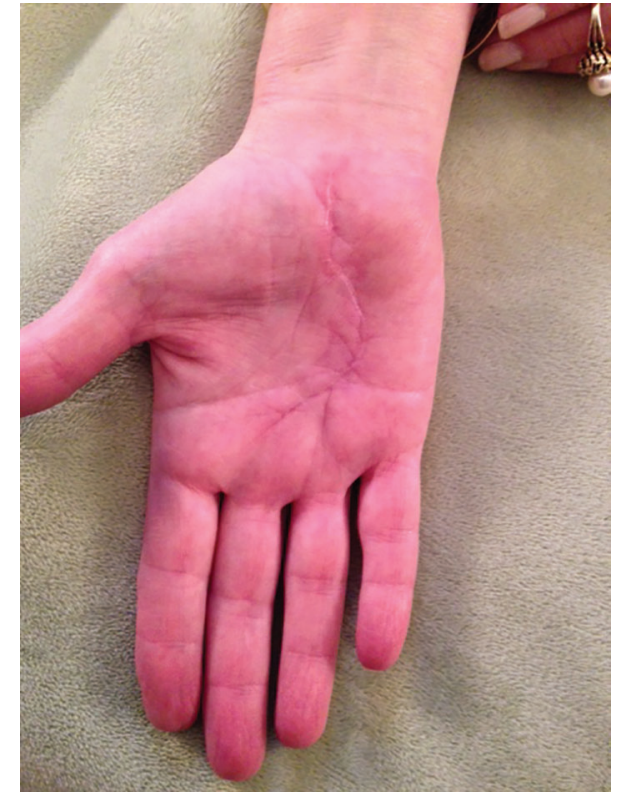

Figure 3 | Post-operative photo after removal of giant palmar lipoma.

\section{References}

1. Bancroft LW, Kransdorf MJ, Peterson JJ, O’Connor MI. Benign fatty tumors: classification, clinical course, imaging appearance, and treatment. Skeletal Radiol. 2006;35:719-33.

2. Fazilleau F, Williams T, Richou J, Sauleau V, Le Nen D. Median nerve compression in carpal tunnel caused by a giant lipoma. Case Rep Orthop. 2014;2014:654934.

3. Fornage BD, Tassin GB. Sonographic appearances of superficial soft tissue lipomas. J Clin Ultrasound. 1991;19:215-20.

4. Grivas TB, Psarakis SA, Kaspiris A. Liapi G. Giant lipoma of the thenar - case study and contemporary approach to its aetiopathogenicity. Hand (NY) 2009;4:173-6.

5. Nadar MM, Bartoli CR, Kasdan ML. Lipomas of the hand: a review and 13 patient case series. Eplasty. 2010;10:e66.

6. Phalen GS, Kendrick JI, Rodriguez JM. Lipomas of the upper extremity: a series of fifteen tumors in the hand and wrist and six tumors causing nerve compression. Am J Surg. 1971;12:298-306.

7. Cribb GL, Cool WP, Ford DJ, Mangham DC. Giant lipomatous tumours of the hand and forearm. J Hand Surg Br. 2005;30:509-12.

8. Carroll RE, Doyle JR. Lipoma of the hand. J Bone Joint Surg [Am]. 1967;49:581.

9. Paarlberg D, Linscheid RL, Soule EH. Lipomas of the hand. Including a case of lipoblastomatosis in a child. Mayo Clin Proc. 1972;47:121-4.

10. Teh J, Whiteley G. MRI of soft tissue masses of the hand and wrist. Br J Radiol. 2007;80:47-63.

11. Hsu CS, Hentz VR, Yao J. Tumours of the hand. Lancet Oncol. 2007;8:157-66.

12. Ramirez-Montaño L, Lopez RP, Ortiz NS. Giant lipoma of the third finger of the hand. SpringerPlus. 2013;2:164.

13. De La Cruz Monroy MF, Durani P, Offer G). Unusual case of finger lipoma: a case report and literature review. J Plast Reconstr Aesthet Surg. 2015;68:284-6.

14. Patterson, JW. Weedon's skin pathology, 4 th ed. Oxford. Elsevier; 2015. Chapter 25, Tumors of fat; p. 1015-28.

15. Galant J, Martí-Bonmatí L, Sáez F, Soler R, Alcalá-Santaella R, Navarro M. The value of fat-suppressed T2 or STIR sequences in distinguishing lipoma from well-differentiated liposarcoma. Eur Radiol. 2003;13:337-43.

\section{Conclusion}

Lipomas are benign fatty tumors that rarely occur on the hand. Typically, they present as slow-growing masses that may develop symptoms if local neurovascular structures are compressed. Imaging techniques and biopsy aid in diagnosis. The main treatment is surgical excision with long-term follow-up.

\section{Acknowledgements}

We would like to thank Dr. Mitchell Klapper for providing the photos included in this review.
16. Chatterton BD, Moores TS, Datta P, Smith KD. An exceptionally large giant lipoma of the hand. BMJ Case Rep. 2013;2013:bcr2013200206.

17. Munk PL, Lee MJ, Janzen DL, Connell DG, Logan PM, Poon PY, et al. Lipoma and liposarcoma: evaluation using CT and MR imaging. AJR Am J Roentgenol. 1997;169:589-94.

18. Inaparthy P, Southgate GW. Giant lipoma of hand: a case report. Eur J Orthop Surg Traumatol. 2006;16:251-2.

19. Johnson CJ, Pynsent PB, Grimer RJ. Clinical features of soft tissue sarcomas. Ann R Coll Surg Engl. 2001;83:203-5.

20. Rubin BP, Fletcher CD. The cytogenetics of lipomatous tumours. Histopathology. 1997;30:507-11.

21. Patel ME, Silver JW, Lipton DE, Pearlman HS. Lipofibroma of the median nerve in the palm and digits of the hand. J Bone Joint Surg Am. 1979;61:393-7.

22. Razzaghi A, Anastakis DJ. Lipofibromatous hamartoma: review of early diagnosis and treatment. Can J Surg. 2005;48:394-9.

23. Capelastegui A, Astigarraga E, Fernandez-Canton G, Saralegui I, Larena JA, Merino A. Masses and pseudomasses of the hand and wrist: MR findings in 134 cases. Skeletal Radiol. 1999;28:498-507.

24. Höglund M, Muren C, Engkvist 0 . Ultrasound characteristics of five common soft-tissue tumors in the hand and forearm. Act Radiol. 1997;38:348-54.

25. Nguyen V, Choi J, Davis KW. Imaging of wrist masses. Curr Probl Diagn Radiol. 2004;33:147-60.

26. Schmidt I. Irreparable radial nerve palsy due to delayed diagnostic management of a giant lipoma at the proximal forearm resulting in a triple tendon transfer procedure: case report and brief review of literature. Open Orthop J. 2017;11:794-803.

27. Higgs PE, Young VL, Schuster R, Weeks PM. Giant lipomas of the hand and forearm. South Med J. 1993;86:887-90. 